\title{
EDITORIAL
}

\section{Tackling the clinical value conundrum}

\author{
How might the challenges of demonstrating the value of new drugs be approached in the \\ context of growing pressures on health-care budgets?
}

On 18 September, the UK's National Institute for Health and Clinical Excellence (NICE) held a technology appraisal meeting to discuss whether the kinase inhibitor lapatinib (Tyverb; GlaxoSmithKline) should be made available through the UK National Health Service (NHS) for the treatment of advanced breast cancer. Such technology appraisal meetings have been occurring regularly since the start of the decade, but what was unusual about this particular meeting was that it was the first that was open to the general public.

In part, this may reflect the growing public interest in the decisions of health technology assessment (HTA) organizations such as NICE in the wake of decisions not to recommend the use of various novel drugs. For example, draft guidance from NICE on the use of four novel drugs for advanced renal cell cancer, issued in August, which recommended against their use on the basis of insufficient demonstration of their cost-effectiveness, has been heavily criticized by oncologists and patient groups. Indeed, the preliminary judgement from NICE based on discussions at the September meeting - announced in mid-October - was to recommend against the use of lapatinib by the NHS.

Such decisions are increasingly presenting a challenge for companies developing novel drugs. Although sales in the US market - in which achieving reimbursement of the latest therapies is typically considerably easier than in countries such as the UK - are still most likely to be the key factor for the overall commercial success of a drug at present, NICE decisions are perceived to influence reimbursement decisions elsewhere in the world. This is especially the case in countries that lack HTA capabilities themselves.

More broadly, however, as discussed in a news story on p876, it seems that the activities of NICE and other HTA organizations have also contributed to a change in perception of how health-care innovation is assessed and rewarded in the past decade. This is reflected in a growing emphasis on understanding the needs of payers earlier in drug development programmes, and on incorporating the collection of additional data relevant to HTA tools such as quality-adjusted life years (QALYs) in clinical trials.

It is not hard to appreciate the extent of the challenges that HTA organizations face in trying to balance limited health-care budgets with the benefits to patients from the introduction of new (and often more expensive) drugs. So, how might these challenges be addressed, while allowing patients to access such drugs, as well as genuine innovation from developers to be rewarded?

Part of the solution might be to try to tackle deficiencies in measures such as the QALY used by NICE, which has been the focus of some of the criticism of their decisions. For example, the 'rule of thumb' threshold at which NICE will support reimbursement by the NHS is around UK£30,000 per QALY, but this number has not kept pace with inflation. The measurement also does not reflect the underlying severity of the condition, and it lacks consideration of broader benefits; for example, to other parts of the health-care system, such as caregivers. Moreover, cost-effectiveness estimates using QALYs can differ widely depending on the underlying methods and assumptions. And a further issue, which is particularly relevant to novel cancer drugs, is that it can be very difficult to demonstrate cost-effectiveness using QALYs in the initial patient population who receive the drug, usually those with the most severe disease.

However, restricting access to such drugs, as in the $\mathrm{UK}$, might mean that the real value of the drug is never demonstrated. More generally, as also highlighted on p876, designing development programmes with too rigid a focus on payer recommendations - at a stage where information on the drug's activity is very limited - could threaten opportunities to understand how to realize its full potential.

In this respect, another emerging approach — risksharing between manufacturers and payers - could represent a potential solution. A pioneering example was provided in 2007, when NICE issued final guidance for the use of bortezomib (Velcade; Janssen-Cilag) for the treatment of multiple myeloma under which the NHS only pays for the drug when patients show a response to treatment (Nature Rev. Drug Discov. 6, 945; 2007). Achieving success with such strategies is also not without major challenges: lapatinib was rejected by NICE even though the manufacturer had proposed a risk-sharing scheme, and the scheme for bortezomib depends on a suitable biomarker of response and presents complex administrative issues. Nevertheless, it still seems to represent one of the best near-term approaches to ensuring that the potential value of innovative drugs is not lost owing to the pressures of cost constraints. 